\title{
Investigation of the Critical Point in Models of the Type of Dyson's Hierarchical Models
}

\author{
P. M. Bleher and Ja. G. Sinai \\ Landau Institute for Theoretical Physics, Academy of Sciences, Moscow, USSR
}

Received March 27, 1973

\begin{abstract}
We consider the classical spin models where the Hamiltonians are small modifications of the Hamiltonians of Dyson's hierarchical models. Under some assumptions we investigate rigorously the neighbourhood of the critical point and find the critical indices. It follows that in the cases under consideration phenomenological Landau's theory of phase transitions is valid.
\end{abstract}

\section{Introduction}

In classical lattice ferromagnets the critical temperature $T_{c r}$ separates the domains with zero and non-zero spontaneous magnetization. The behaviour of different thermodynamical parameters near $T_{c r}$ was considered rigorously for the two-dimensional Ising model using Onsager's exact solution (see [1,2]), and some other models (see [3]) also using the exact formula for the free energy.

Recently Dyson introduced so-called hierarchical models immitating in many respects the lattice systems with pairwise long-range power interaction. Under several natural assumptions Dyson proved that the spontaneous magnetization in his models is non-zero for sufficiently large $\beta([4-6])$.

We consider in this paper a class of models slightly generalizing hierarchical models and find rigorously under some conditions critical indices for them. Recently some non-rigorous results in this direction were obtained by Baker [7]. Our case corresponds to the "gaussian case" of his paper. Closely related results were presented also in [8] (see also [9]).

Now we want to describe briefly our main results. We impose certain conditions on the distribution of the mean spin in a finite volume of fixed size for an interval of temperatures. Under these conditions we prove the existence of a critical temperature $T_{c r}$ inside this interval and for $T=T_{c r}$ we establish the limit distribution for the mean spin which is a gaussian distribution with a non-usual normalization. This permits us to find the asymptotic of binary correlation functions for $T=T_{c r}$.

For $T>T_{c r}$ we obtain the asymptotic expression for the susceptibility and binary correlation functions. For $T<T_{c r}$ we find an asymptotic 
expression for the spontaneous magnetization. The critical indices are just as predicted by Landau's theory $([10,9])$.

The case $T=T_{c r}$ was considered by both authors. The cases $T<T_{c r}$, $T>T_{c r}$ were considered by the first author.

\section{Hierarchical Models and Asymptotically-Hierarchical Models}

We begin with the description of general hierarchical models. Assume to be given a sequence of positive integers $r_{n}, r_{n} \geqq 2$, $n=1, \ldots, q_{n}=\prod_{i=1}^{n} r_{i}$, and for any integer $r \geqq 2$ some positive quadratic form $Q_{r}\left(t_{1}, \ldots, t_{r}\right)$. In general hierarchical models one considers the sequence of volumes $V_{n}=V_{n, 0}$ in which each volume $V_{n, 0}$ consists of $q_{n}$ points. The volume $V_{n, 0}$ is decomposed into $r_{n}$ subvolumes $V_{n-1, i}$, $i=1, \ldots, r_{n}$, each of which consists of $q_{n-1}$ points; the subvolume $V_{n-1, i}$ is decomposed into $r_{n-1}$ subvolumes $V_{n-2, i}$ each of which consists of $q_{n-2}$ points, and so on. For any $k \geqq 0$ the volume $V_{n, 0}$ is decomposed into $r_{n} \cdot \ldots \cdot r_{n-k}$ subvolumes $V_{n-k-1, i}$ each of which consists of $q_{n-k-1}$ points. The Hamiltonian is defined by a sequence of positive numbers $b_{p}$ and has the form

$$
H_{n}=-\sum_{p=1}^{n} b_{p} \sum_{i=0, \bigcup_{k=1}^{p_{p}} \sum_{p-1, i_{k}}^{q_{n} / q_{p}-1} V_{p, i}}^{V_{r_{p}}}\left(s_{i_{1}}, s_{i_{2}}, \ldots, s_{i_{r_{p}}}\right) .
$$

Here $s_{i_{l}}$ is the mean spin in the volume $V_{p-1, i_{l}}$. We assume that the spin $s_{x}, x \in V_{n, 0}$ takes values \pm 1 .

Dyson ([4-6]) considered the case $r_{n} \equiv 2$ and $Q_{2}\left(t_{1}, t_{2}\right)=\left(\frac{t_{1}+t_{2}}{2}\right)^{2}$. Baker considered the cases $r_{n} \equiv 2,4,8$ ([7]).

We shall consider below slightly more general models which we shall call asymptotically-hierarchical models (a.h.m.). The Hamiltonian in these models is defined by an integer $n_{0}$ and can be written for $n>n_{0}$ in the following form

$$
H_{n}=-\sum_{i=1}^{q_{n} / q_{n_{0}}} H_{n_{0}}\left(S_{n_{0}, i}\right)-\sum_{p=n_{0}+1}^{n} b_{p} \sum_{\substack{i, r_{p} \\ k=1}} \sum_{p-1, l_{k}=V_{p, i}} Q_{r_{p}}\left(s_{i_{1}}, \ldots, s_{i_{r_{p}}}\right) .
$$

Here $S_{n_{0}, i}$ is the configuration of spins in $V_{n_{0}, i}$ and $H_{n_{0}}$ is an arbitrary Hamiltonian in the volume of $q_{n_{0}}$ points, satisfying the symmetry condition: $H_{n_{0}}\left(S_{n_{0}, i}\right)=H_{n_{0}}\left(-S_{n_{0}, i}\right)$.

In the sequel only the case $r_{n} \equiv r$ and $b_{p}=c^{p}$ will be discussed. Moreover we shall assume that the quadratic form $Q_{r}\left(t_{1}, \ldots, t_{r}\right)$ is symmetric 
in its arguments and has the form

$$
Q_{r}\left(t_{1}, \ldots, t_{r}\right)=g_{r}\left(\frac{t_{1}+\cdots+t_{r}}{r}\right)^{2}+h_{r}\left(\frac{t_{1}^{2}+\cdots+t_{r}^{2}}{r}\right) ;
$$

we shall assume that $g_{r}=g, h_{r}=h, h+g>0, r g+c h>0$. The extension of our results to other cases will be done in another paper.

For a.h.m. the limit free energy per particle $\chi$ exists and is a convex function of its arguments if $c<r$. An extension of Dyson's arguments shows that spontaneous magnetization for sufficiently large $\beta$ is nonzero if $c>1$.

\section{Formulation of Results}

For a.h.m. let us denote $g_{k}(s ; \beta)=\operatorname{Prob}_{k}\left(s_{k, 0}=s \mid \beta\right)$, where $\operatorname{Prob}_{k}(\cdot \mid \beta)$ means the Gibbs probability distribution with parameter $\beta$ and zero magnetic field in the volume $V_{k, 0}$.

For $k>n_{0}$ one can write the following recurrence relations:

$$
\begin{aligned}
g_{k}(s ; \beta)= & \frac{\Xi_{k-1}^{r}(\beta)}{\Xi_{k}(\beta)} \\
& \cdot \sum_{\frac{s_{1}+\cdots+s_{r}}{r}=s} \exp \left(\beta c^{k-1} Q\left(s_{1}, s_{2}, \ldots, s_{r}\right)\right) g_{k-1}\left(s_{1} ; \beta\right) \ldots g_{k-1}\left(s_{r} ; \beta\right)
\end{aligned}
$$

where $\Xi_{k(\beta)}$ is the grand partition function in the volume $V_{k, 0}$. For all $k$ the probabilities $g_{k}(s ; \beta)$ are even functions of $s$.

Let us introduce some normalization for spins $s_{k, 0}$ putting $c^{\frac{k}{2}} s_{k, 0}=z_{k}$. It is evident that $z_{k}$ takes the values $\frac{-r^{k}+2 m}{r^{k}} \cdot c^{\frac{k}{2}}, 0 \leqq m \leqq r^{k}$. Therefore for $\Delta_{k}=\frac{c^{\frac{k}{2}}}{r^{k}}$ and $f_{k}(z ; \beta)=g_{k}\left(z c^{-\frac{k}{2}} ; \beta\right)$ we have instead of (1)

$$
\begin{aligned}
f_{k}(z ; \beta)= & L_{k} \sum_{\frac{z_{1}+\ldots+z_{r}}{r}=\frac{z}{\sqrt{\bar{c}}}} \exp \left(\beta Q\left(z_{1}, \ldots, z_{r}\right)\right) \\
& \cdot f_{k-1}\left(z_{1} ; \beta\right) \cdot \ldots \cdot f_{k-1}\left(z_{r} ; \beta\right) \Delta_{k-1}^{r-1} .
\end{aligned}
$$

Here $L_{k}$ is a normalization constant.

It is natural to assume that for large $k$ the behaviour of the functions $f_{k}$ is the same as the behaviour of the iterates of the non-linear integral transformation

$$
Q_{\beta}(f)=\frac{\sum_{i=1}^{r} z_{i}=\frac{r}{\sqrt{c}} z}{\int \exp \left(\beta Q\left(z_{1}, \ldots, z_{r}\right) f\left(z_{1}\right) \cdot \ldots \cdot f\left(z_{r}\right) \prod_{i} d z_{i}\right.} .
$$


The investigation of such transformations begins with the search for the fixed points or the eigenvectors, i.e. the functions $f$ which satisfy the equality

$$
f(z)=\lambda \int_{\sum z_{\imath}=\frac{r}{\sqrt{c}} z} \exp \left(\beta Q\left(z_{1}, \ldots, z_{r}\right)\right) f\left(z_{1}\right) \cdot \ldots \cdot f\left(z_{r}\right) \prod_{i} d z_{i}=\tilde{Q}_{\beta}(f)
$$

for some constant $\lambda$. Having such a fixed point it is necessary to investigate its stability properties. Only fixed points with some stability properties can appear as limits of our difference transformations (2).

It is easy to verify that the function $\exp \left(-a_{0}(\beta) z^{2}\right)$ satisfies the Eq. (3) with $a_{0}(\beta)=\frac{g+h}{r-c} \beta$, i.e. it is an eigenfunction of the transformation $\tilde{Q}_{\beta}$. Thus the function $\sqrt{\frac{a_{0}}{\pi}} \exp \left(-a_{0}(\beta) z^{2}\right)=e_{\beta}(z)$ is a fixed point for the transformation $Q_{\beta}$.

To investigate the stability properties of $e_{\beta}(z)$ let us consider now the differential of the transformation $Q_{\beta}$ near the point $e_{\beta}(z)$. It is easier to consider the differential of the transformation $\tilde{\tilde{Q}}_{\beta}$ near the point $e_{\beta}(z)$. Taking $e(z)=e_{\beta}(z)+\varepsilon h(z)$ one finds easily

$$
\begin{aligned}
\mathscr{D} \tilde{Q}_{\beta} h= & \left.\frac{d}{d \varepsilon} \tilde{Q}_{\beta}\left(e_{\beta}+\varepsilon h\right)\right|_{\varepsilon=0} \\
= & \frac{d}{d \varepsilon}\left(\frac{a_{0}}{\pi}\right)^{\frac{r}{2}} \int_{\sum z_{i}=\frac{r}{\sqrt{c}} z} \exp \left(\beta Q\left(z_{1}, \ldots, z_{r}\right)\right)\left(e_{\beta}\left(z_{1}\right)+\varepsilon h\left(z_{1}\right)\right) \\
& \cdot \ldots \cdot\left(e_{\beta}\left(z_{r}\right)+\varepsilon h\left(z_{r}\right)\right) \prod_{i} d z_{i} \\
= & \exp \left(-a_{0} z^{2}\right) r\left(\frac{\zeta(\beta)}{\pi}\right)^{\frac{1}{2}} \int_{-\infty}^{+\infty} \exp \left(\zeta(\beta)\left(\frac{z}{\sqrt{c}}-z_{1}\right)^{2}+a_{0}(\beta) z_{1}^{2}\right) \\
& \cdot h\left(z_{1}\right) d z_{1}
\end{aligned}
$$

where

$$
\zeta(\beta)=\frac{h \cdot \beta-\mathrm{a}_{0}(\beta) r}{r-1},
$$

which is the well-known integral transformation with Gaussian kernel (see [11]). This operator is a compact selfadjoint operator in the Hilbert space $\mathscr{H}\left(\gamma_{0}\right)=L_{\text {ev }}^{2}\left(\mathbb{R}^{1} ; e^{-\gamma_{0} z^{2}}\right)$ of real valued even square-integrable functions with the weight $e^{-\gamma_{0} z^{2}}$, where $\gamma_{0}=\gamma_{0}(\beta)=\zeta(\beta)\left(1-\frac{1}{c}\right)+2 a_{0}(\beta)$; the spectrum of this integral operator in the space $\mathscr{H}\left(\gamma_{0}\right)$ consists of the infinite sequence of numbers $r, \frac{r}{c}, \frac{r}{c^{2}}, \frac{r}{c^{3}}, \ldots$ The corresponding 
eigenfunctions are $\left\{e^{-a_{0}(\beta) z^{2}} G_{2 i}(z ; \gamma)\right\}_{i=0}^{\infty}$,

where

$$
\gamma=\gamma(\beta)=\zeta(\beta)\left(1-\frac{1}{c}\right)
$$

$$
G_{2 i}(z ; \gamma)=\sqrt[4]{\frac{\gamma}{\pi}} \frac{\sqrt{(2 i) !}}{2^{i}} \sum_{j=0}^{i} \frac{(-1)^{i-j}(2 \sqrt{\gamma} z)^{2 j}}{(2 j) !(i-j) !}
$$

is the $2 i$-th Hermite polynomial.

The first eigenvalue $r$ is determined by the fact that our transformation is $r$-linear. The second eigenvalue is always more than one. If $c>\sqrt{r}$ then all other eigenvalues are less than one. We shall show that in an a.h.m. the convergence of $f_{k}(z ; \beta)$ to a gaussian distribution for $\beta=\beta_{c r}$ is in general possible if $c>\sqrt{r}$. Thus we impose the first important condition.

Condition 1. $c>\sqrt{r}$.

Now we are going to discuss our next condition. As the reader will see its exact formulation is not too short. Therefore we want to explain its meaning. One can hope that $f_{n}(z ; \beta)$ for $\beta=\beta_{c r}$ will tend to $e_{\beta_{c r}}(z)$ if the functions $f_{n_{0}}\left(z ; \beta_{c r}\right)$ are sufficiently close to $e_{\beta_{c r}}(z)$ in an appropriate sense for some sufficiently large $n=n_{0}$. Here we give the exact formulation of what we mean by "sufficiently close" for our problem. The number $n_{0}=n_{0}(c, Q)$ depends only on the constant $c$ and the quadratic form $Q$. Its exact value is defined by some number (near ten) of inequalities appearing during the proof. Therefore we shall not give its explicit expression here.

Furthermore, if we are given a family of probability distributions $f_{n_{0}}(z ; \beta)$ for some interval of temperatures $\boldsymbol{\beta}=\left[\beta^{-}, \beta^{+}\right]$we don't know the value of the critical temperature without solving the whole sequence of recurrence Eqs. (2). However it is possible to formulate conditions which guarantee that the critical temperature will lie inside the interval $\boldsymbol{\beta}$.

Now we proceed to the exact formulation.

Condition 2. Let us choose three numbers $0<\varrho, q, \xi<1$ depending only on $c$ in such a way that

$$
\begin{gathered}
\frac{r}{c^{2}}<\varrho<1 ; \\
\frac{r^{\frac{1}{2}}}{c^{1^{3 / 4}}}, \frac{r^{2}}{c^{4}}<q<\frac{r}{c^{2}} ; \\
c^{-\frac{1}{3}}, q^{\frac{1}{4}}, \frac{q c^{2}}{r}<\xi<1 .
\end{gathered}
$$

There exist a number $n_{0}(c)$, an interval of inverse temperatures $\boldsymbol{\beta}_{n_{0}}=\left[\beta_{n_{0}}^{-}, \beta_{n_{0}}^{+}\right]$, and a differentiable function $b_{n_{0}}(\beta)=b(\beta)$, defined on 
this interval, the number $d$ satisfying the inequalities

$$
0<d, r+1<d_{1}^{2} \text { for } d_{1}=\frac{d(\sqrt{c}-1)}{2 \sqrt{c}},
$$

for which

$$
\begin{aligned}
\left.\mathrm{a}_{1}\right) & b\left(\beta_{n_{0}}^{-}\right)=-\left(\frac{c}{2} \varrho\right)^{n_{0}}, b\left(\beta_{n_{0}}^{+}\right)=\left(\frac{c}{2} \varrho\right)^{n_{0}},|b(\beta)|<\left(\frac{c}{2} \varrho\right)^{n_{0}} \text { for } \\
& \beta \in\left(\beta_{n_{0}}^{-}, \beta_{n_{0}}^{+}\right) .
\end{aligned}
$$

$\mathrm{a}_{2}$ ) for each $\beta \in \boldsymbol{\beta}_{n_{0}}$ the function $f_{n_{0}}(z ; \beta)$ can be represented in the following form

$$
f_{n_{0}}(z ; \beta)=L_{n_{0}}(\beta) \exp \left(-\left(a_{0}(\beta)+\left(\frac{2}{c}\right)^{n_{0}} b(\beta)\right) z^{2}\right)\left(1+q_{n_{0}}(z ; \beta)\right)
$$

with $L_{n_{0}}(\beta)$ being a constant factor (with respect to $z$ ) and for the "small perturbation" $q_{n_{0}}(z ; \beta)$ satisfying

$\left.\mathrm{a}_{2}^{\prime}\right)$ for $|z| \leqq \frac{d \sqrt{n_{0}}}{\sqrt{a_{0}}}$

$$
q_{n_{0}}(z ; \beta)=\delta_{n_{0}}(\beta) G_{4}(z ; \gamma)+R_{n_{0}}(z ; \beta)
$$

with $\gamma=\gamma(\beta)=\zeta(\beta)\left(1-\frac{1}{c}\right)$ and $\delta_{n_{0}}(\beta), \quad R_{n_{0}}(z ; \beta)$ are differentiable functions of $\beta \in \boldsymbol{\beta}_{n_{0}}$,

$$
\begin{gathered}
\left|R_{n_{0}}(z ; \beta)\right| \leqq q^{n_{0}} \leqq-\delta_{n_{0}} \xi^{n_{0}}, \\
-\delta_{n_{0}} \leqq q^{\frac{n_{0}}{2}} \xi^{n_{0}} .
\end{gathered}
$$

$\left.\mathrm{a}_{2}^{\prime \prime}\right)$ for $|z|>\frac{d \sqrt{n_{0}}}{\sqrt{a_{0}}}$

$$
\begin{gathered}
0 \leqq 1+q_{n_{0}}(z ; \beta) \leqq \exp \left(-v_{n_{0}} z^{4}\right), \\
0<v_{n_{0}}<-0,1 \cdot \delta_{n_{0}} .
\end{gathered}
$$

Remark. The proof of Theorem 1 below shows that the Condition 2 is stable in the sense that it is fulfilled for $n>n_{0}$ if it is fulfilled for $n=n_{0}$.

Now we can give the exact formulation of our theorems.

Theorem 1. If the Conditions 1, 2 hold, then there exists one and only one $\beta_{c r} \in \boldsymbol{\beta}_{n_{0}}$ for which

$$
\lim _{n \rightarrow \infty} \operatorname{Prob}_{n}\left\{t_{1}<s_{n, 0} c^{\frac{n}{2}}<t_{2} ; \beta_{c r}\right\}=\frac{1}{\sqrt{2 \pi \sigma}} \int_{t_{1}}^{t_{2}} e^{-\frac{u^{2}}{2 \sigma}} d u
$$

for some positive $\sigma$ and arbitrary $t_{1}, t_{2}$. 
Theorem 1 shows that for $\beta=\beta_{c r}$ the mean spin $s_{n, 0}$ has gaussian distribution with non-usual normalization.

Corollary 1. For $\beta=\beta_{c r}$ the binary correlation function $\left\langle s_{x}, s_{y}\right\rangle_{n, \beta_{c r}}$, $x, y \in V_{n, 0}$ satisfies the inequalities

$$
C_{1} \cdot c^{-d(x, y)} \leqq\left\langle s_{x}, s_{y}\right\rangle_{n, \beta_{c r}} \leqq C_{2} c^{-d(x, y)}
$$

with some constants $C_{1}, C_{2}$, where $d(x, y)$ is the least number $k$ such that $x, y \in V_{k, i}$ for some $i$.

Theorem 2. Under the conditions of Theorem 1 let $\beta_{n_{0}}^{+}<\beta<\beta_{c r}$. Then for the mean spin $s_{n, 0}$

$$
\lim _{n \rightarrow \infty} \operatorname{Prob}_{n}\left\{t_{1}<s_{n, 0} r^{\frac{n}{2}}<t_{2} ; \beta\right\}=\frac{1}{\sqrt{2 \pi \sigma_{1}(\beta)}} \int_{t_{1}}^{t_{2}} e^{-\frac{u^{2}}{2 \sigma_{1}(\beta)}} d u
$$

where $\sigma_{1}(\beta) \sim \operatorname{const}\left(\beta_{c r}-\beta\right)^{-1}$.

Corollary 2. Under the conditions of Theorem 2 the binary correlation functions $\left\langle s_{x}, s_{y}\right\rangle_{n, \beta}, x, y \in V_{n, 0}$ satisfy for all $n$ the inequalities

$$
\frac{C_{1}^{\prime}}{\left(\beta_{c r}-\beta\right)^{2}}\left(\frac{r^{2}}{c}\right)^{-d(x, y)} \leqq\left\langle s_{x}, s_{y}\right\rangle_{n, \beta} \leqq \frac{C_{2}^{\prime}}{\left(\beta_{c r}-\beta\right)^{2}}\left(\frac{r^{2}}{c}\right)^{-d(x, y)}
$$

with some constants $C_{1}^{\prime}, C_{2}^{\prime}$.

Theorem 3. Under the conditions of Theorem 1 let $\beta_{c r}<\beta<\beta_{n_{0}}^{-}$. Then there exist positive functions $m(\beta), \sigma_{2}(\beta), m(\beta) \sim \operatorname{const}\left(\beta-\beta_{c r}\right)^{\frac{1}{2}}$, $\sigma_{2}(\beta) \sim \operatorname{const}\left(\beta-\beta_{c r}\right)$, such that for a sequence $m_{n}(\beta), \lim _{n \rightarrow \infty} m_{n}(\beta)=m(\beta)$,

$$
\begin{aligned}
& \lim _{n \rightarrow \infty} \operatorname{Prob}_{n}\left\{t_{1}<\left(s_{n, 0}-m_{n}(\beta)\right) r^{\frac{n}{2}}<t_{2} ; \beta\right\}=\frac{1}{2} \frac{1}{\sqrt{2 \pi \sigma_{2}(\beta)}} \int_{t_{1}}^{t_{2}} e^{-\frac{u^{2}}{2 \sigma_{2}(\beta)}} d u, \\
& \lim _{n \rightarrow \infty} \operatorname{Prob}_{n}\left\{t_{1}<\left(s_{n, 0}+m_{n}(\beta)\right) r^{\frac{n}{2}}<t_{2} ; \beta\right\}=\frac{1}{2} \frac{1}{\sqrt{2 \pi \sigma_{2}(\sigma)}} \int_{t_{1}}^{t_{2}} e^{-\frac{u^{2}}{2 \sigma_{2}(\beta)}} d u .
\end{aligned}
$$

\section{Thermodynamical Limit of Hierarchical and Asymptotically-Hierarchical Models}

Let $V$ be a countable set and $r_{n}$ be a sequence of integers, $r_{n}>1$. Following Vershik let us say that a hierarchical $\left\{r_{n}\right\}$-structure is defined on $V$ if there is defined a decreasing sequence $\xi_{1} \geqq \xi_{2} \geqq \cdots$ of partitions of $V$ with the following properties:

$\left.\mathrm{a}_{1}\right) \xi_{1}=\varepsilon$ where $\varepsilon$ is as usual the partition of $V$ into separate points;

$\mathrm{a}_{2}$ ) any element of $\xi_{i}$ consists of $r_{i}$ elements of $\xi_{i-1}$;

$\mathrm{a}_{3}$ ) for any two points $x, y \in V$ there exists a number $d(x, y)$ such that $x, y$ belong to the same element of any partition $\xi_{i}, i \geqq d(x, y)$. 
In the following $d(x, y)$ will be the least number with this property. The number $\prod_{i=1}^{d(x, y)} r_{i}$ plays the role of a distance between $x, y$. All spaces with $\left\{r_{n}\right\}$-structure are naturally isomorphic.

If $V$ is the space with $\left\{r_{n}\right\}$-structure then $V^{(k)}=V \mid \xi_{k}$ is the space with $\left\{r_{n}^{\prime}\right\}$-structure where $r_{n}^{\prime}=r_{n+k}$.

Let us denote by $G=G(V)$ the group of all finite permutations of $V$ leaving each $\xi_{i}$ invariant, and let $\Omega(V)\left(\Omega_{0}(V)\right)$ be the space of all realvalued $( \pm 1$-valued) functions on $V$. We can define in the usual way the probability distributions on $\Omega(V)$ and $\Omega_{0}(V)$, gaussian probability distributions on $\Omega(V)$, the distributions invariant under the group $G$, and so on.

Now let us return to the definition of Dyson's hierarchical models. One can consider the volumes $V_{n, 0}$ as an increasing sequence of subsets of the infinite space $V$ with $\left\{r_{n}\right\}$-structure such that $\bigcup_{n=1}^{\infty} V_{n, 0}=V$. The sequence of probability distributions $\operatorname{Prob}_{n}(\cdot \mid \beta)$ can be considered as a sequence of probability distributions on $\Omega_{0}(V)$ defined on an increasing sequence of corresponding $\sigma$-algebras. Dyson in [4] proved in fact the following theorem.

Theorem 4. For any $\beta>0$ the sequence of probability distributions $\operatorname{Prob}_{n}(\cdot \mid \beta)$ converges in a natural sense to a limit Gibbsian distribution $P(\cdot \mid \beta)$ defined on the $\sigma$-algebra of measurable subsets of $\Omega_{0}(V)$ and invariant under the group $G$.

For the asymptotically-hierarchical models the same considerations lead to the following theorem.

Theorem 5. Under the conditions of Theorem 1 the probability distributions $\operatorname{Prob}_{n}(\cdot \mid \beta)$ converge in a natural way to a limit Gibbsian distribution $P(\cdot \mid \beta)$. The distribution on $\Omega\left(V^{\left(n_{0}\right)}\right)=\Omega\left(V \mid \xi_{n_{0}}\right)$ induced by a map

$$
\pi_{n_{0}}: \Omega_{0}(V) \rightarrow \Omega\left(V_{n_{0}}\right) \text { where } \pi_{n_{0}}(f)(x)=\frac{1}{\prod_{i=1}^{n_{0}} r_{i}} \sum_{y \in x} f(y)
$$

is invariant under the corresponding group $G$.

Now we shall formulate the results concerning the limit distributions $P(\cdot \mid \beta)$ which are equivalent to the Theorems 1, 2, 3. Let be $s_{n}=\frac{1}{r^{n}} \sum_{x \in C_{n}} s(x)$ where $C_{n}$ is an arbitrary element of the partition $\xi_{n}$ entering into the definition of a hierarchical structure on $V$. We shall consider the distribution of $s_{n}$ which depends only on $n$ but not on $C_{n}$. 
Theorem 1'. Under the conditions of Theorem 1 for $\beta=\beta_{c r}$ and arbitrary fixed $t_{1}, t_{2}$

$$
\lim _{n \rightarrow \infty} \mathrm{P}\left\{t_{1}<s_{n} \cdot c^{\frac{n}{2}}<t_{2} ; \beta_{c r}\right\}=\frac{1}{\sqrt{2 \pi \sigma^{\prime}}} \int_{t_{1}}^{t_{2}} e^{-\frac{u^{2}}{2 \sigma^{\prime}}} d u
$$

for some positive constant $\sigma^{\prime}$.

Corollary 1'. There exist constants $C_{1}, C_{2}$ such that for $\beta=\beta_{c r}$

$$
C_{1} c^{-d(x, y)} \leqq\left\langle s_{x}, s_{y}\right\rangle_{\beta_{c r}} \leqq C_{2} c^{-d(x, y)} \text {. }
$$

Theorem 2'. Under the conditions of Theorems 1 and 2 for arbitrary fixed $t_{1}, t_{2}$

$$
\lim _{n \rightarrow \infty} \mathrm{P}\left\{t_{1}<s_{n} r^{n}<t_{2} ; \beta\right\}=\frac{1}{\sqrt{2 \pi \sigma_{1}^{\prime}(\beta)}} \int_{t_{1}}^{t_{2}} e^{-\frac{u^{2}}{2 \sigma_{1}^{\prime}(\beta)}} d u
$$

where $\sigma_{1}^{\prime}(\beta) \sim \operatorname{const}\left(\beta_{c r}-\beta\right)^{-1}$.

Corollary 2'. Under the conditions of Theorem 2 the binary correlations functions $\left\langle s_{x}, s_{y}\right\rangle_{\beta}, x, y \in V$ satisfy the inequalities

$$
\frac{C_{1}^{\prime}}{\beta_{c r}-\beta} r^{-d(x, y)} \leqq\left\langle s_{x}, s_{y}\right\rangle_{\beta} \leqq \frac{C_{2}^{\prime}}{\beta_{c r}-\beta} r^{-d(x, y)}
$$

with some constants $C_{1}^{\prime}, C_{2}^{\prime}$.

Theorem 3'. In the notation of Theorem 3 under the conditions of the Theorem 1 for $\beta_{c r}<\beta<\beta_{n_{0}}^{-}$and arbitrary $t_{1}, t_{2}$

$$
\lim _{n \rightarrow \infty} \mathrm{P}\left\{t_{1}<\left(s_{n}-m_{n}(\beta)\right) r^{\frac{n}{2}}<t_{2}\right\}=\frac{1}{2} \frac{1}{\sqrt{2 \pi \sigma_{2}^{\prime}(\beta)}} \int_{t_{1}}^{t_{2}} e^{-\frac{u^{2}}{2 \sigma_{2}^{\prime}(\beta)}} d u
$$

where $\sigma_{2}^{\prime}(\beta) \sim \operatorname{const}\left(\beta-\beta_{c r}\right)^{-1}$.

\section{Proof of Theorem 1}

We shall consider only the case $r=2$ and $Q\left(z_{1}, z_{2}\right)=c\left(\frac{z_{1}+z_{2}}{2}\right)^{2}$. The generalization to other cases is straight-forward. We shall construct a sequence of imbedded segments $\boldsymbol{\beta}_{n}=\left[\beta_{n}^{-}, \beta_{n}^{+}\right]$and a sequence of differentiable functions $b_{n}(\beta)$ defined on $\boldsymbol{\beta}_{n}$ such that for all $\beta \in \boldsymbol{\beta}_{n}$ the functions $f_{n}(z ; \beta)$ can be represented in the following form

$$
f_{n}(z ; \beta)=L_{n}(\beta) \exp \left[-\left(a_{0}(\beta)+\left(\frac{2}{c}\right)^{n} b_{n}(\beta)\right) z^{2}\right]\left(1+q_{n}(z ; \beta)\right)
$$


where $L_{n}(\beta), b_{n}(\beta), q_{n}(z ; \beta)$ satisfy some estimates listed below. From these estimates it follows that

$$
\left(\frac{2}{c}\right)^{n} b_{n}(\beta) \underset{n \rightarrow \infty}{\longrightarrow} 0 ; 0 \leqq 1+q_{n}(z ; \beta) \leqq 2 \text { for } \beta \in \boldsymbol{\beta}_{n}
$$

and $-\infty<z<\infty$;

$$
q_{n}(z ; \beta) \underset{n \rightarrow \infty}{\longrightarrow} 0 \text { uniformly for }|z|<\frac{d \sqrt{n}}{\sqrt{a_{0}}},
$$

$\beta \in \boldsymbol{\beta}_{n}, d$ is a constant depending only on $c, L_{n}(\beta) \rightarrow L=\sqrt{\frac{a_{0}(\beta)}{\pi}}$. Thus we get the assertion of Theorem 1 for $\beta_{c r}=\bigcap_{n} \boldsymbol{\beta}_{n}$.

The representation (5) is non-unique because one can change $b_{n}$ or $L_{n}$ and include the difference in $q_{n}$ without changing $f_{n}$. The most crucial part of the proof is the special choice of $L_{n}$ and $b_{n}$. This will be described precisely during the proof.

The variable $z$ in (5) varies from $-c^{\frac{n}{2}}$ to $c^{\frac{n}{2}}$ with the step $\Delta_{n}=2 c^{\frac{n}{2}} \cdot 2^{-n}$. The set of values of $z$ will be denoted as $M_{n}$.

The substitution of (5) into the formula (2) gives (one should remember that $\left.r=2, Q\left(z_{1}, z_{2}\right)=c\left(\frac{z_{1}+z_{2}}{2}\right)^{2}\right)$

$$
\begin{gathered}
f_{n+1}(z ; \beta)=\tilde{L}_{n+1} \exp \left(-\tilde{\lambda}_{n+1} z^{2}\right) \sqrt{\frac{2 \lambda_{n}}{\pi}} \sum_{u} e^{-2 \lambda_{n} u^{2}}\left(1+q_{n}\left(\frac{z}{\sqrt{c}}-u ; \beta\right)\right) \\
\cdot\left(1+q_{n}\left(\frac{z}{\sqrt{c}}+u ; \beta\right)\right) \Delta_{n}
\end{gathered}
$$

where

$$
\begin{gathered}
\tilde{\lambda}_{n+1}=\tilde{\lambda}_{n+1}(\beta)=a_{0}(\beta)+\left(\frac{2}{c}\right)^{n+1} b_{n}(\beta), \\
\lambda_{n}=\lambda_{n}(\beta)=a_{0}(\beta)+\left(\frac{2}{c}\right)^{n} b_{n}(\beta), \Delta_{n}=2 c^{\frac{n}{2}} 2^{-n} .
\end{gathered}
$$

The summation goes over $u$ for which $\frac{z}{\sqrt{c}}-u \in M_{n}, \frac{z}{\sqrt{c}}+u \in M_{n}$ and $z \in M_{n+1}$. Let us put

$$
\begin{array}{r}
1+\tilde{q}_{n+1}(z ; \beta)=\sqrt{\frac{2 \lambda_{n}}{\pi}} \sum_{u} e^{-2 \lambda_{n} u^{2}}\left(1+q_{n}\left(\frac{z}{\sqrt{c}}-u ; \beta\right)\right) \\
\cdot\left(1+q_{n}\left(\frac{z}{\sqrt{c}}+u ; \beta\right)\right) \Delta_{n} .
\end{array}
$$


If we introduce the linear integral operator

$$
\mathscr{A}_{n} q=2 \sqrt{\frac{2 \lambda_{n}}{\pi}} \int_{-\infty}^{+\infty} e^{-2 \lambda_{n} u^{2}} q\left(\frac{z}{\sqrt{c}}-u\right) d u
$$

and assume, that the function $q_{n}(z ; \beta)$ is extended to the whole line $\mathbb{R}^{1}$ as a nice function of $z \in \mathbb{R}^{1}$ then we can rewrite (7) in the following way

$$
\tilde{q}_{n+1}=\mathscr{A}_{n} q_{n}+S_{n}\left(q_{n}\right)
$$

where $S_{n}\left(q_{n}\right)$ will be treated as a remainder term. In fact $S_{n}\left(q_{n}\right)$ is the sum of two terms. The first one appears from the non-linear part of the formula (7):

$$
S_{n}^{(1)}\left(q_{n}\right)=\sqrt{\frac{2 \lambda_{n}}{\pi}} \sum_{u} e^{-2 \lambda_{n} u^{2}} q_{n}\left(\frac{z}{\sqrt{c}}-u ; \beta\right) q_{n}\left(\frac{z}{\sqrt{c}}+u ; \beta\right) \Delta_{n} .
$$

The second term $S_{n}^{(2)}\left(q_{n}\right)$ has the form

$$
S_{n}^{(2)}\left(q_{n}\right)=\left(\tilde{\mathscr{A}}_{n}-\mathscr{A}_{n}\right)\left(q_{n}+\frac{1}{2}\right) \text {, }
$$

where

$$
\tilde{\mathscr{A}_{n}} q=2 \sqrt{\frac{2 \lambda_{n}}{\pi}} \sum_{u} e^{-2 \lambda_{n} u^{2}} q_{n}\left(\frac{z}{\sqrt{c}}-u ; \beta\right) \Delta_{n}
$$

These properties of $\mathscr{A}_{n}$ can be easily verified:

$1^{\circ} . \mathscr{A}_{n}$ is a self-adjoint compact operator in the Hilbert space $\mathscr{H}\left(\gamma_{n}\right)=L_{\mathrm{ev}}^{2}\left(\mathbb{R}^{1} ; e^{-\gamma_{n} z^{2}}\right)$ of real-valued even square-integrable functions with the weight $e^{-\gamma_{n} z^{2}}, \gamma_{n}=2 \lambda_{n}\left(1-\frac{1}{c}\right)$.

$2^{\circ} . \mathscr{A}_{n} \mathbb{1}=2 \cdot \mathbb{1}$.

$3^{\circ}$. The spectrum of $\mathscr{A}_{n}$ in the space $\mathscr{H}\left(\gamma_{n}\right)$ consist of the numbers $\left\{\frac{2}{c^{j}}\right\}_{j=0}^{\infty} ;$ the corresponding eigenfunctions are even Hermite polynomials $G_{2 j}\left(z ; \gamma_{n}\right), j=0,1, \ldots$

$4^{\circ} .\left\|\mathscr{A}_{n} q\right\|_{C} \leqq 2\|q\|_{C}$

$$
\left\|\mathscr{A}_{n} q\right\|_{C^{1}} \leqq K\|q\|_{C}
$$

where $K$ is some constant.

From $3^{\circ}$ it follows that two eigenvalues $2,2 \cdot c^{-1}$ are always greater than 1 ; the others are smaller than one because of the condition $c>\sqrt{2}$. The main idea is to choose $b_{n}(\beta)$ and $L_{n}(\beta)$ in such a way that the projections of $q_{n}(z ; \beta)$ on the expanding subspaces $\mathscr{H}_{\text {exp }}\left(\gamma_{n}\right)$ generated by eigenvectors $G_{0}\left(z ; \gamma_{n}\right), G_{2}\left(z ; \gamma_{n}\right)$ are equal to zero or at least very small. The possibility of doing so for $n=n_{0}$ follows easily from the Condition 2 . 
Let us fix $\omega=\omega(c)>1$ so that $\omega-1$ is sufficiently small and consider the sequence $n_{i}=\left[n_{0} \omega^{i}\right], \quad i=0,1, \ldots$. We shall prove that for $n=n_{0}, n_{1}, n_{2}, \ldots$, the representations

$$
f_{n}(z ; \beta)=L_{n}(\beta) \exp \left(-\left(\mathrm{a}_{0}(\beta)+\left(\frac{2}{c}\right)^{n} b_{n}(\beta)\right) z^{2}\right)\left(1+q_{n}(z ; \beta)\right)
$$

can be chosen in such a way that

1) $L_{n}(\beta), b_{n}(\beta)$ are differentiable functions of $\beta \in \boldsymbol{\beta}_{n}$,

$$
b_{n}\left(\beta_{n}^{-}\right)=-\left(\frac{c}{2} \varrho\right)^{n}, b_{n}\left(\beta_{n}^{+}\right)=\left(\frac{c}{2} \varrho\right)^{n},\left|b_{n}(\beta)\right| \leqq\left(\frac{c}{2} \varrho\right)^{n}, \beta \in \boldsymbol{\beta}_{n} .
$$

2) $q_{n}(z ; \beta), z \in M_{n},|z|<\frac{d \sqrt{n}}{\sqrt{a_{0}}}$, can be represented in the form

$$
q_{n}(z ; \beta)=\delta_{n} G_{4}\left(z ; \gamma_{n}\right)+R_{n}(z ; \beta)
$$

where $\delta_{n}=\delta_{n}(\beta)$ is a differentiable function of $\beta \in \boldsymbol{\beta}_{n}$ and

$$
K_{n}^{(1)}\left(\frac{2}{c^{2}}\right)^{n-n_{0}}<-\delta_{n}<K_{n}^{(2)}\left(\frac{2}{c^{2}}\right)^{n-n_{0}}
$$

where $K_{n}^{(i)}=\delta_{n_{0}}\left(\prod_{j=n_{0}}^{n}\left(1+\alpha^{j}\right)\right)^{(-1)^{i}}, i=1,2,0<\alpha=\alpha(c)<1, R_{n}(z ; \beta)$ is a differentiable function of $\beta \in \boldsymbol{\beta}_{n}$,

$$
\left|R_{n}(z ; \beta)\right|<q^{n} .
$$

3) for $|z|>\frac{d \sqrt{n}}{\sqrt{a_{0}}}$

$$
0 \leqq 1+q_{n}(z ; \beta) \leqq \exp \left(-v_{n} z^{4}\right)
$$

with

$$
v_{n}=v_{n_{0}}\left(\frac{2}{c^{2}}\right)^{n-n_{0}} \prod_{j=n_{0}}^{n}\left(1+\alpha^{j}\right)^{-1} .
$$

In order to use the contractive properties of $\mathscr{A}_{n}$ we shall employ a more detailed representation of $q_{n}(z ; \beta)$ for $n_{i}<n \leqq n_{i+1}$. Namely, let us write

2') for $|z|<\frac{d \sqrt{n}}{\sqrt{a_{0}}}$

$$
q_{n}(z ; \beta)=\sum_{j=2}^{N} \delta_{n}^{(j)} G_{2 j}\left(z ; \gamma_{n}\right)+H_{n}(z ; \beta)+T_{n}(z ; \beta)
$$


where $N=N(c)$ is so big that

$$
\begin{gathered}
\xi^{N}<\frac{1}{2},\left(\frac{2}{c_{0}^{N}}\right)^{\omega-1} e^{d^{2}}<\frac{q}{2}, \quad c_{0}=\xi c>1 ; \\
K_{n}^{(1)}\left(\frac{2}{c^{2}}\right)^{n}<-\delta_{n}^{(2)}<K_{n}^{(2)}\left(\frac{2}{c^{2}}\right)^{n}, \\
\left|\delta_{n}^{(j)}\right|<2 q^{n_{2}}\left(\frac{2}{c_{0}^{j}}\right)^{n-n_{2}-1}, \quad j=3, \ldots, N .
\end{gathered}
$$

The function $H_{n}(z ; \beta)$ is extended to the whole segment

$$
D_{n}=\left[-\frac{d \sqrt{n}}{\sqrt{a_{0}}}, \frac{d \sqrt{n}}{\sqrt{a_{0}}}\right]
$$

as a $C^{1}$-function and $H_{n}(z ; \beta)=0$ for $z \notin D_{n}$ and

$$
\left\|\sum_{j=2}^{N} \delta_{n}^{(j)} G_{2 j}\left(z ; \gamma_{n}\right)+H_{n}(z ; \beta)\right\|_{C^{m}\left(D_{n}\right)} \leqq K_{m} 3^{n-n_{l}} q^{n_{\imath}},
$$

$m=0,1, K_{m}=K_{m}(c)$ is a constant,

$$
\begin{gathered}
\left(H_{n}, G_{2 j}\right)_{\mathscr{H}\left(\gamma_{n}\right)}=\int_{z \in D_{n}} H_{n}(z ; \beta) G_{2 j}\left(z ; \gamma_{n}\right) e^{-\gamma_{n} z^{2}} d z=0, \quad j=0,1, \ldots, N, \\
\left\|H_{n}\right\|_{\mathscr{H}\left(\gamma_{n}\right)} \leqq 2 q^{n_{i}}\left(\frac{2}{c^{N+1}}\right)^{n-n_{i}-1} .
\end{gathered}
$$

All the functions $\delta_{n}^{(j)}=\delta_{n}^{(j)}(\beta), 2 \leqq j \leqq N, H_{n}(z ; \beta), T_{n}(z ; \beta)$ are differentiable functions of $\beta \in \boldsymbol{\beta}_{n}$ and

$$
\left|T_{n}(z ; \beta)\right|<(\xi q)^{n_{2+1}} 3^{n-n_{2+1}} .
$$

It is very important that in 2), 2') the main role is played by the projection of $q_{n}$ on the third eigenspace generated by $G_{4}\left(z ; \gamma_{n}\right)$ and that this projection is negative. From this fact it follows that $1+q_{n}(z ; \beta)$ has the form drawn in Fig. 1:

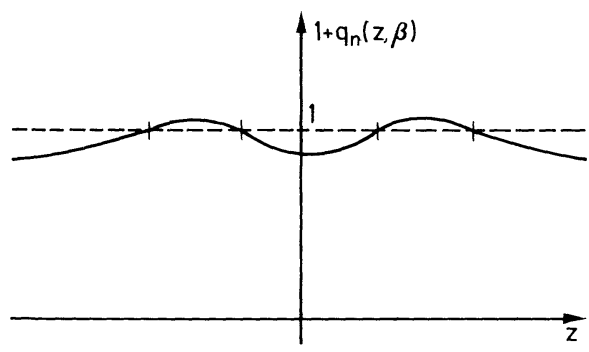

Fig. 1 
Let us denote by $\left(\mathscr{U}_{n}\right)$ the properties 1$\left.\left.), 2\right), 3\right)$ and by $\left(\mathscr{V}_{n}\right)$ the properties 1), 2'), 3). Now Theorem 1 will follow from the next three lemmas. large.

Lemma 1. $\left(\mathscr{V}_{n}\right)$ implies $\left(\mathscr{U}_{n}\right)$ for $n=n_{i+1}$ if $N=N(c)$ is sufficiently

Lemma 2. For $n_{i}<n \leqq n_{i+1}, q_{n+1}$ can be chosen in such a way that $\left(\mathscr{V}_{n}\right)$ implies $\left(\mathscr{V}_{n+1}\right)$.

Lemma 3. For $n=n_{i}, q_{n+1}$ can be chosen in such a way that $\left(\mathscr{U}_{n}\right)$ implies $\left(\mathscr{V}_{n+1}\right)$.

Proof of Lemma 1. Only the implication $\left.2^{\prime}\right) \Rightarrow 2$ ) must be proved. From $2^{\prime}$ ) one has

$$
\begin{gathered}
\int_{z \in D_{n}}\left|H_{n}(z ; \beta)\right|^{2} d z \leqq e^{d^{2} n} \int_{z \in D_{n}}\left|H_{n}(z ; \beta)\right|^{2} e^{-\gamma_{n} z^{2}} d z ; \\
\left\|H_{n}(z ; \beta)\right\|_{C^{1}\left(D_{n}\right)} \leqq\left\|\sum_{j=2}^{N} \delta_{n}^{(j)} G_{2 j}\left(z ; \gamma_{n}\right)+H_{n}(z ; \beta)\right\|_{C^{1}\left(D_{n}\right)} \\
+\sum_{j=2}^{N}\left|\delta_{n}^{(j)}\right|\left\|G_{2 j}\left(z ; \gamma_{n}\right)\right\|_{C^{1}\left(D_{n}\right)} \leqq 1,
\end{gathered}
$$

because $N=N(c)$ is fixed, $\left\|G_{2 j}(z ; \beta)\right\|_{C^{1}\left(D_{n}\right)}$ increase as some power of $n$, $\left|\delta_{n}^{(j)}\right|$ decrease as a geometric progression and $n_{0}$ can be assumed to be sufficiently large.

Let us introduce the function $\varphi(z)$ where

$$
\varphi(z)= \begin{cases}1+z & -1 \leqq z \leqq 0 \\ 0 & \text { for other } z\end{cases}
$$

From the inequality $\left|\frac{d}{d z} H_{n}(z ; \beta)\right|<1$ one has

$$
\left|H_{n}(z ; \beta)\right| \geqq\left|H_{n}\left(z_{0} ; \beta\right) \varphi\left(z-z_{0}\right)\right|
$$

for any $z_{0}>0, z_{0}<\frac{d \sqrt{n}}{\sqrt{a_{0}}}$. This gives

$$
\left\|H_{n}(z ; \beta)\right\|_{L^{2}} \geqq\left|H_{n}\left(z_{0} ; \beta\right)\right|\left\|\varphi\left(z-z_{0}\right)\right\|_{L^{2}}=\sqrt{\frac{1}{3}}\left|H_{n}\left(z_{0} ; \beta\right)\right| .
$$

Now the desired estimate for the $C\left(D_{n}\right)$-norm of $H_{n}(z ; \beta)$ follows from the $\mathscr{H}\left(\gamma_{n}\right)$-norm of $H_{n}(z ; \beta)$. Other terms in $R_{n}(z ; \beta)$ have good $C$-norms, as can be seen directly from $\left.2^{\prime}\right){ }^{\circ}$ Thus we have the desired estimate for the $C$-norm of $R_{n}(z ; \beta)$. Q.E.D.

Proof of Lemma 2. We shall use an important local property of the expression (7) for $1+\tilde{q}_{n+1}(z ; \beta)$. Namely, the part of the sum in (7) for $u$, $|u|>\frac{d_{1} \sqrt{n}}{\sqrt{a_{0}}}$, with some constant $d_{1}=d_{1}(c)$ gives a value which is less 
then $\varepsilon=5 e^{-d_{1}^{2} n}$ because from $\left.2^{\prime}\right)$ and 3 ) one has $0 \leqq 1+q_{n}(z ; \beta) \leqq 2$. Therefore $\tilde{q}_{n+1}\left(z_{0} ; \beta\right)$ depends mainly on the values of $q_{n}(z ; \beta)$ for $\left|z-\frac{z_{0}}{\sqrt{c}}\right|<\frac{d_{1} \sqrt{n}}{\sqrt{a_{0}}}$ with $\varepsilon$-error. In particular for

$$
z \in D_{n+1}=\left[-\frac{d \sqrt{n+1}}{\sqrt{a_{0}}}, \frac{d \sqrt{n+1}}{\sqrt{a_{0}}}\right]
$$

$\tilde{q}_{n+1}(z ; \beta)$ is defined with $\varepsilon$-error by the values of $q_{n}(z ; \beta)$ for $z \in D_{n}$ because it follows from the Condition 2 that

$$
1-\frac{1}{\sqrt{c}}>\frac{d_{1}}{d} \text {. }
$$

Now we proceed to construct $q_{n+1}(z ; \beta)$. First of all let us include the part of the sum in (7) with $|u| \geqq \frac{d_{1} \sqrt{n}}{\sqrt{a_{0}}}$ in $T_{n+1}(z ; \beta)$. If $d_{1}$ is sufficiently large this term will satisfy the estimates for $T_{n+1}$.

Now we shall estimate the remainder term $S_{n}\left(q_{n}\right)=S_{n}^{(1)}\left(q_{n}\right)+S_{n}^{(2)}\left(q_{n}\right)$ [see (9)]. Due to the quadratic character of $S_{n}^{(1)}\left(q_{n}\right)$ one has

$$
\left\|S_{n}^{(1)}\left(q_{n}\right)\right\|_{C\left(D_{n+1}\right)} \leqq 1,1 \cdot\left\|q_{n}\right\|_{C\left(D_{n}\right)}^{2} .
$$

This inequality shows that $S_{n}^{(1)}\left(q_{n}\right)$ can be also included in $T_{n+1}(z ; \beta)$ if the estimate of $\left\|q_{n}\right\|_{C\left(D_{n}\right)}^{2}$ which follows from $\left.2^{\prime}\right)$ is better than the estimate of $T_{n+1}(z ; \beta)$ in $\left.2^{\prime}\right)$. The last assertion is true when $\omega-1$ is sufficiently small.

Let us denote

$$
\begin{aligned}
& B_{n} q(z)=2 \sqrt{\frac{2 \lambda_{n}}{\pi}} \int_{u \in D_{n}} e^{-2 \lambda_{n} u^{2}} q\left(\frac{z}{\sqrt{c}}-u\right) d u, \\
& \tilde{B}_{n} q(z)=2 \sqrt{\frac{2 \lambda_{n}}{\pi}} \sum_{u \in D_{n}} e^{-2 \lambda_{n} u^{2}} q\left(\frac{z}{\sqrt{c}}-u\right) \Delta n .
\end{aligned}
$$

It is easy to see that

$$
\left\|\tilde{B}_{n} q\right\|_{C} \leqq 2,1\|q\|_{C} .
$$

So we can include the term $\tilde{B}_{n} T_{n}(z ; \beta)$ into $T_{n+1}(z ; \beta)$. Next we consider instead of the function $\tilde{B}_{n}\left(\frac{1}{2}+\sum_{j=2}^{N} \delta_{n}^{(j)} G_{2 j}\left(z ; \gamma_{n}\right)+H_{n}(z ; \beta)\right)$ the function $\mathscr{A}_{n}\left(\frac{1}{2}+\sum_{j=2}^{N} \delta_{n}^{(j)} G_{2 j}\left(z ; \gamma_{n}\right)+H_{n}(z ; \beta)\right)$. The error can be estimated using the $C^{1}$-estimate of $\left.2^{\prime}\right)$ of the function $\sum_{j=2}^{N} \delta_{n}^{(j)} G_{2 j}\left(z ; \gamma_{n}\right)+H_{n}(z ; \beta)$ and the 
following inequalities:

$$
\left\|\left(\tilde{B}_{n}-B_{n}\right) q\right\|_{C} \leqq\|q\|_{C^{1}} \Delta_{n}
$$

(which follow from a simple interpolation formula) and

$$
\begin{aligned}
\left\|\left(\mathscr{A}_{n}-\mathscr{B}_{n}\right) q\right\|_{C} & =\left\|2 \sqrt{\frac{2 \lambda_{n}}{\pi}} \quad \int_{|u| \geqq \frac{d_{1} \sqrt{n}}{\sqrt{a_{0}}}} e^{-2 \lambda_{n} u^{2}} q\left(\frac{z}{\sqrt{c}}-u\right) d u\right\|_{C} \\
& \leqq 2\|q\|_{C} e^{-d_{1}^{2} n} .
\end{aligned}
$$

Due to these inequalities we include the error into $T_{n+1}(z ; \beta)$. From the property $3^{\circ}$ of the operator $\mathscr{A}_{n}$ it follows that $\mathscr{A}_{n}\left(\frac{1}{2}\right)=1$ and

$$
\begin{aligned}
\mathscr{A}_{n}\left(\sum_{j=2}^{N} \delta_{n}^{(j)} G_{2 j}\left(z ; \gamma_{n}\right)+H_{n}(z ; \beta)\right) & =\sum_{j=2}^{N} \delta_{n}^{(j)} \frac{2}{c^{j}} G_{2 j}\left(z ; \gamma_{n}\right) \\
\quad+\mathscr{A}_{n} H_{n}(z ; \beta), \quad H_{n} \perp G_{2 j} & \text { in } \mathscr{H}\left(\gamma_{n}\right), \quad j=0,1, \ldots, N,
\end{aligned}
$$

and consequently

$$
\left\|\mathscr{A}_{n} H_{n}\right\|_{\mathscr{H}\left(\gamma_{n}\right)} \leqq \frac{2}{c^{N+1}}\left\|H_{n}\right\|_{\mathscr{H}\left(\gamma_{n}\right)} .
$$

It follows from the last estimate that

$$
\mathscr{A}_{n} H_{n}(z ; \beta)=\sum_{j=0}^{N} \tilde{\delta}_{n+1}^{(j)} G_{2 j}\left(z ; \gamma_{n}\right)+\tilde{H}_{n+1}(z ; \beta)
$$

where $\tilde{H}_{n+1}(z ; \beta)$ is a smooth function of $z \in D_{n+1}, \tilde{H}_{n+1}(z ; \beta)=0$ for

$$
\begin{gathered}
z \notin D_{n+1},\left(\tilde{H}_{n+1}, G_{2 j}\right)_{\mathscr{H}\left(\gamma_{n}\right)}=0, j=0,1, \ldots, N,\left|\tilde{\delta}_{n+1}^{(j)}\right|, \\
\left\|\tilde{H}_{n+1}\right\|_{\mathscr{H}\left(\gamma_{n}\right)} \leqq \frac{2}{c^{N}}\left\|H_{n}\right\|_{\mathscr{H}\left(\gamma_{n}\right)} .
\end{gathered}
$$

Therefore denoting $\tilde{\tilde{\delta}}_{n+1}^{(j)}=\frac{2}{c^{j}} \delta_{n}^{(j)}+\tilde{\delta}_{n+1}^{(j)}$ we have

$$
\begin{aligned}
1+\tilde{q}_{n+1}(z ; \beta)=1 & +\sum_{j=0}^{1} \tilde{\delta}_{n+1}^{(j)} G_{2 j}\left(z ; \gamma_{n}\right)+\sum_{j=2}^{N} \tilde{\tilde{\delta}}_{n+1}^{(j)} G_{2 j}\left(z ; \gamma_{n}\right) \\
& +\tilde{H}_{n+1}(z ; \beta)+\tilde{T}_{n+1}(z ; \beta),
\end{aligned}
$$

where $\tilde{\delta}_{n+1}^{(j)}, \tilde{H}_{n+1}, \tilde{T}_{n+1}$ satisfy $2^{\prime}$ ). Let us put

$$
\gamma_{n+1}=2\left(a_{0}(\beta)+\left(\frac{2}{c}\right)^{n+1} b_{n}(\beta)\right)\left(1-\frac{1}{c}\right)
$$

and rewrite the last equality putting $\gamma_{n+1}$ instead of $\gamma_{n}$

$$
\begin{aligned}
1+\tilde{q}_{n+1}(z ; \beta)=1 & +\sum_{j=0}^{1} \delta_{n+1}^{(j)} G_{2 j}\left(z ; \gamma_{n+1}\right)+\sum_{j=2}^{N} \delta_{n+1}^{(j)} G_{2 j}\left(z ; \gamma_{n+1}\right) \\
& +H_{n+1}(z ; \beta)+T_{n+1}(z ; \beta),
\end{aligned}
$$


where $\delta_{n+1}^{(j)}, j=2, \ldots, N, H_{n+1}, T_{n+1}$ also satisfy $2^{\prime}$ ) because

$\left|\gamma_{n}-\gamma_{n+1}\right|<\left(\frac{2}{c}\right)^{n+1}\left|b_{n}(\beta)\right|<\frac{2}{c} \varrho^{n}, \quad\left|\delta_{n+1}^{(j)}\right|<\left|\gamma_{n}-\gamma_{n+1}\right|\left|\delta_{n}^{(2)}\right| \leqq\left(\frac{2 \varrho}{c^{2}}\right)^{n}$,

For $z \in D_{n+1}$

$$
j=0,1 \text {. }
$$

$$
1+\sum_{j=0}^{1} \delta_{n+1}^{(j)} G_{2 j}\left(z ; \gamma_{n+1}\right)=e^{\sum_{j=0}^{1} \delta_{n+1}^{(j)} G_{2 j}\left(z ; \gamma_{n+1}\right)}+T_{n+1}^{(1)}(z ; \beta)
$$

where $T_{n+1}^{(1)}$ satisfies the estimate of $T_{n+1}$ in $2^{\prime}$ ). Now we can define $q_{n+1}$ from the expression

$$
1+q_{n+1}(z ; \beta)=e^{-\sum_{j=0}^{1} \delta_{n+1}^{(j)} G_{2 J}\left(z ; \gamma_{n+1}\right)}\left(1+\tilde{q}_{n+1}(z ; \beta)\right) .
$$

It follows from the above estimates that with this definition of $q_{n+1}(z ; \beta)$ $\left.2^{\prime}\right)$ is true.

The function $\sum_{j=0}^{1} \delta_{n+1}^{(j)} G_{2 j}\left(z ; \gamma_{n+1}\right)$ is the even quadratic polynomial of $z$. Thus we have the representation (5) for $f_{n+1}(z ; \beta)$ where the Gaussian multiplier is

$$
\begin{array}{r}
\tilde{L}_{n+1}(\beta) \exp \left(-\left(a_{0}(\beta)+\left(\frac{2}{c}\right)^{n+1} b_{n}(\beta)\right) z^{2}+\sum_{j=0}^{1} \delta_{n+1}^{(j)} G_{2 j}\left(z ; \gamma_{n+1}\right)\right) \\
=L_{n+1}(\beta) \exp \left(-\left(a_{0}(\beta)+\left(\frac{2}{c}\right)^{n+1} b_{n+1}(\beta)\right) z^{2}\right) .
\end{array}
$$

The last expression gives also the definition of the $b_{n+1}(\beta)$. It follows that

$$
\left(\frac{2}{c}\right)^{n+1}\left|b_{n}(\beta)-b_{n+1}(\beta)\right|<K\left(\frac{2 \varrho}{c^{2}}\right)^{n}, \quad K=K\left(c, \beta_{n_{0}}^{-}, \beta_{n_{0}}^{+}\right)
$$

is a constant. Therefore

$$
\left(\frac{2}{c}\right)^{n+1} b_{n+1}\left(\beta_{n}^{+}\right)>\left(\frac{2}{c}\right)^{n+1} b_{n}\left(\beta_{n}^{+}\right)-K\left(\frac{2 \varrho}{c^{2}}\right)^{n}=\varrho^{n}\left(\frac{2}{c}-K\left(\frac{2}{c^{2}}\right)^{n}\right)>\varrho^{n}
$$

and in a similar way $\left(\frac{2}{c}\right)^{n+1} b_{n+1}\left(\beta_{n}^{-}\right)<-\varrho^{n}$. Furthermore $b_{n+1}(\beta)$ is a differentiable function of $\beta$ and we can find a segment $\beta_{n+1}=\left[\beta_{n+1}^{-}, \beta_{n+1}^{+}\right] \subset \boldsymbol{\beta}_{n}$ satisfying 1$)$.

Next we consider the estimate 3 ) for $1+q_{n+1}(z ; \beta)$. It can be easily seen from 2) that the estimate 3 ) is true not only for $|z|>\frac{d \sqrt{n}}{\sqrt{a}}$ but also 
for $|z|>d_{2}$ where $d_{2}$ is some constant not depending on $n$. Therefore $\left\{z: 1+q_{n+1}(z ; \beta)>1\right\} \subset D=\left[-d_{2}, d_{2}\right]$ and we can write for all $z$

$$
0 \leqq 1+q_{n}(z ; \beta) \leqq e^{-v_{n} z^{4}}\left(1+\mu_{n} \chi_{D}(z)\right)
$$

where $v_{n}=v_{n_{0}}\left(\frac{2}{c^{2}}\right)^{n-n_{0}} \prod_{j=n_{0}}^{n}\left(1+\alpha^{j}\right)^{-1}, \mu_{n}=2\left|\delta_{n}^{(2)}\right|$, and $\chi_{D}$ is the indicator of $D$. Substitution of the last inequality into (7) gives

$$
\begin{aligned}
1+ & \tilde{q}_{n+1}(z ; \beta)<\sqrt{\frac{2 \lambda_{n}}{\pi}} \sum_{u} e^{-2 \lambda_{n} u^{2}} e^{-v_{n}\left(\frac{2}{c^{2}} z^{4}+\frac{12}{c} z^{2} u^{2}+2 u^{4}\right)} \\
& \cdot\left(1+\mu_{n} \chi_{D}\left(\frac{z}{\sqrt{c}}-u\right)\left(1+\mu_{n} \chi_{D}\left(\frac{z}{\sqrt{c}}+u\right)=a_{1}+a_{2} \mu_{n}+a_{3} \mu_{n}^{2} .\right.\right.
\end{aligned}
$$

It is easy to see that for

$$
0 \leqq a_{1}<\exp \left(-v_{n} \frac{2}{c^{2}} z^{4}\right)\left(1-\frac{K_{0}}{n+1}\right)
$$

where $K_{0}$ is a constant not depending on $n$,

$$
0 \leqq a_{2}<K_{1} \exp \left(-v_{n} \frac{2}{c^{2}} z^{4}\right)
$$

$a_{3}=0$ because $\chi_{D}\left(\frac{z}{\sqrt{c}}-u\right) \chi_{D}\left(\frac{z}{\sqrt{c}}+u\right)=0$ for $|z|>\frac{d_{2} \sqrt{n+1}}{\sqrt{a_{0}}}$.

This gives

$$
\begin{aligned}
0 & \leqq 1+\tilde{q}_{n+1}(z ; \beta) \leqq \exp \left(-v_{n} \frac{2}{c^{2}} z^{4}\right)\left(1-\frac{K_{0}}{n+1}+K_{1} \mu_{n}\right) \\
& \leqq \exp \left(-\frac{2}{c^{2}} v_{n} z^{4}\right) .
\end{aligned}
$$

Now 3$)$ for $1+q_{n+1}(z ; \beta)$ is a simple consequence of the last inequality. Lemma 2 is proved.

Proof of Lemma 3. We rewrite the formula (7) in the following way

$$
\tilde{q}_{n+1}(z ; \beta)=\tilde{\mathscr{A}}_{n} q_{n}(z ; \beta)+S_{n}^{(1)}\left(q_{n}\right)(z ; \beta)
$$

where

$$
\tilde{\mathscr{A}}_{n} q(z)=2 \sqrt{\frac{2 \lambda_{n}}{\pi}} \sum_{u} e^{-2 \lambda_{n}\left(\frac{z}{\sqrt{c}}-u\right)^{2}} q(u) \Delta_{n}
$$

is the linear part of the transformation (7) and $S_{n}^{(1)}\left(q_{n}\right)$ defined in (9), is the nonlinear part of this transformation. One can easily verify the following properties of the operator $\tilde{\mathscr{A}_{n}}$ : 
$1^{\circ}$. The formula (14) is meaningful for all $z \in \mathbb{R}^{1}$ even if $q_{n}(z ; \beta)$ is defined only for $z \in M_{n}$ and

$$
\begin{aligned}
\left\|\tilde{\mathscr{A}}_{n} q\right\|_{C\left(\mathbb{R}^{1}\right)} & <3\|q\|_{C\left(M_{n}\right)} \\
\left\|\tilde{\mathscr{A}}_{n} q\right\|_{C^{1}\left(\mathbb{R}^{1}\right)} & <K\|q\|_{C\left(M_{n}\right)} \quad K \text { is a constant }
\end{aligned}
$$

not depending on $q$ and $n$.

$$
\begin{aligned}
& 2^{\circ} \text {. For }|z|<\frac{d \sqrt{n+1}}{\sqrt{a_{0}}} \\
& \tilde{\mathscr{A}}_{n} G_{4}\left(z ; \gamma_{n}\right)=\mathscr{A}_{n} G_{4}\left(z ; \gamma_{n}\right)+\left(\tilde{\mathscr{A}_{n}}-\mathscr{A}_{n}\right) G_{4}\left(z ; \gamma_{n}\right)=\frac{2}{c^{2}} G_{4}\left(z ; \gamma_{n}\right)+T_{n+1}^{(1)}(z ; \beta)
\end{aligned}
$$

where $\mathscr{A}_{n}$ is the integral operator, defined in (8) and $T_{n+1}^{(1)}(z ; \beta)$ satisfies the estimate of the $T_{n+1}(z ; \beta)$ in $\left.2^{\prime}\right)$.

It follows from these properties of the operator $\tilde{\mathscr{A}}_{n}$ and from the estimate of $S_{n}^{(1)}\left(q_{n}\right)(z ; \beta)$ during the proof of Lemma 2 that

$$
\tilde{q}_{n+1}(z ; \beta)=\frac{2}{c^{2}} \delta_{n} G_{4}\left(z ; \gamma_{n}\right)+\tilde{\mathscr{A}}_{n} R_{n}(z ; \beta)+T_{n+1}(z ; \beta)
$$

where the sum $\frac{2}{c^{2}} \delta_{n} G_{4}\left(z ; \gamma_{n}\right)+\tilde{\mathscr{A}_{n}} R_{n}(z ; \beta)$ satisfies the $C^{m}$-estimate, $m=0,1$, of $\left.2^{\prime}\right)$ because of property $1^{\circ}$ of the operator $\tilde{\mathscr{A}_{n}}$ and $T_{n+1}(z ; \beta)$ satisfies the corresponding estimate of $2^{\prime}$ ) too. Next we decompose the sum $\frac{2}{c^{2}} \delta_{n} G_{4}\left(z ; \gamma_{n}\right)+\tilde{\mathscr{A}}_{n} R_{n}(z ; \beta)$ (this decomposition is unique):

$$
\frac{2}{c^{2}} \delta_{n} G_{4}\left(z ; \gamma_{n}\right)+\tilde{\mathscr{A}_{n}} R_{n}(z ; \beta)=\sum_{j=0}^{N} \delta_{n+1}^{(j)} G_{2 j}\left(z ; \gamma_{n+1}\right)+H_{n+1}(z ; \beta)
$$

where $H_{n+1}(z ; \beta)=0$ for $|z|>\frac{d \sqrt{n+1}}{\sqrt{a_{0}}}$ and

$$
\begin{gathered}
\left(H_{n+1}, G_{2 j}\right)_{\mathscr{H}\left(\gamma_{n+1}\right)}=\int_{z \in D_{n+1}} H_{n+1}(z ; \beta) G_{2 j}\left(z ; \gamma_{n+1}\right) e^{-\gamma_{n+1} z^{2}} d z=0, \\
j=0,1, \ldots, N .
\end{gathered}
$$

The desired estimates of $\delta_{n+1}^{(j)}, j=2, \ldots, N$ and $H_{n+1}(z ; \beta)$ of $\left.2^{\prime}\right)$ follow from the $C$-estimate of the $R_{n}(z ; \beta)$ of 2$)$ and from the property $1^{\circ}$ of the operator $\tilde{\mathscr{A}}_{n}$. At last we annul the projections $\delta_{n+1}^{(j)} G_{j}\left(z ; \gamma_{n+1}\right), j=0,1$, in the formula (15) on the expanding eigenvectors of the operator $\mathscr{A}_{n+1}$ in the same way as in the proof of the Lemma 2, i.e. changing a little the Gaussian multiplier in the representation (5). One can see that for $q_{n+1}(z ; \beta)$ all the estimates of $\left.2^{\prime}\right)$ are true. The proof of the properties 1) and 3$)$ of the function $q_{n+1}(z ; \beta)$ is just the same as we used during the proof of Lemma 2. This completes the proof of Lemma 1 and also the proof of Theorem 1 .

Proofs of Theorems 2, 3 will be published in another paper. 
Acknowledgments. The authors express their gratitude to Professors R. L. Dobrushin, O. Lanford, I. M. Lifshitz, R. A. Minlos for valuable discussions. We thank very much Prof. Lanford for his help in preparing this text for publication.

\title{
References
}

1. Onsager, L.: Phys. Rev. 65, 117 (1944)

2. Huang, K.: Statistical Mechanics. New York-London: John Wiley and Sons Inc. 1963

3. Lieb,E., Mattis, D.: Mathematical Physics in one dimension. New York: Academic Press 1966

4. Dyson, F.: Commun. math. Phys. 12, 91 (1969)

5. Dyson, F.: Commun. math. Phys. 12, 212 (1969)

6. Dyson, F.: Commun. math. Phys. 21, (4), 269-283 (1971)

7. Baker,Jr. G. A.: Phys. Rev. B5, 2622 (1972)

8. Fisher, M., Shang-Keng Ma, Nickel, .: Phys. Rev. Letters 29, (4) 917 (1972)

9. Wilson, K., Kogut,I.: The Renormalization group and $\varepsilon$-expansion. Institute for Advanced Studies, Princeton, July, 1972

10. Kadanoff, L. et al.: Rev. Mod. Phys. 39, 395 (1967)

11. Bateman, G., Erdelyi, A.: Higher transcendental functions, Vol. II. New York, Toronto, London: McGraw-Hill Book Company 1955

\author{
P. M. Bleher \\ Ja. G. Sinai \\ Landau Institute \\ for Theoretical Physics \\ Academy of Sciences \\ Vorobiev Chausse, 2 \\ Moscow V-233, USSR
}

